\title{
Characterization and Analysis of Whole Transcriptome of Giant Panda Spleens: Implying Critical Roles of Long Non- Coding RNAs in Immunity
}

\author{
Rui Peng ${ }^{a} \quad$ Yuliang Liu ${ }^{b}$ Zhigang Cai ${ }^{b}$ Fujun Shen ${ }^{b}$ Jiasong Chen ${ }^{a}$ Rong Hou \\ Fangdong Zou ${ }^{\mathrm{a}}$
}

aKey Laboratory of Bio-resources and Eco-environment (Ministry of Education), College of Life Sciences, Sichuan University, Chengdu, 'Sichuan Key Laboratory of Conservation Biology for Endangered Wildlife, Chengdu Research Base of Giant Panda Breeding, Chengdu, Sichuan, China

\section{Key Words \\ Ailuropoda melanoleuca $•$ Transcriptome $・$ Non-coding RNA $・$ Immune response}

\begin{abstract}
:
Background/Aims: Giant pandas, an endangered species, are a powerful symbol of species conservation. Giant pandas may suffer from a variety of diseases. Owing to their highly specialized diet of bamboo, giant pandas are thought to have a relatively weak ability to resist diseases. The spleen is the largest organ in the lymphatic system. However, there is little known about giant panda spleen at a molecular level. Thus, clarifying the regulatory mechanisms of spleen could help us further understand the immune system of the giant panda as well as its conservation. Methods: The two giant panda spleens were from two male individuals, one newborn and one an adult, in a non-pathological condition. The whole transcriptomes of mRNA, IncRNA, miRNA, and circRNA in the two spleens were sequenced using the Illumina HiSeq platform. EBseq and IDEG6 were used to observe the differentially expressed genes (DEGs) between these two spleens. Gene Ontology and KEGG analyses were used to annotate the function of DEGs. Furthermore, networks between non-coding RNAs and protein-coding genes were constructed to investigate the relationship between noncoding RNAs and immune-associated genes. Results: By comparative analysis of the whole transcriptomes of these two spleens, we found that one of the major roles of IncRNAs could be involved in the regulation of immune responses of giant panda spleens. In addition, our results also revealed that microRNAs and circRNAs may have evolved to regulate a large set of biological processes of giant panda spleens, and circRNAs may function as miRNA sponges. Conclusion: To our knowledge, this is the first report of IncRNAs and circRNAs in giant panda, which could be a useful resource for further giant panda research. Our study reveals the potential functional roles of miRNAs, IncRNAs, and circRNAs in giant panda spleen.
\end{abstract}

R. Peng and Y. Liu contributed equally to this work. 


\section{Introduction}

The giant panda, Ailuropoda melanoleuca, is a world-renowned endangered mammal indigenous to China. Like other mammals, giant pandas suffer from a variety of diseases including infectious or communicable diseases, which are seriously threatening the species, especially captive populations [1]. For example, the introduction of invasive Escherichia coli 0152 from a wild panda resulted in the death of about 20 captive giant pandas in the 1980s (unpublished data). In 2015, there was an outbreak of canine distemper virus (CDV) infections among endangered giant pandas, and five of six CDV-infected animals died [2].

The spleen is the largest organ of the lymphatic system and plays important roles in the immune system. The spleen removes older erythrocytes, recycles iron, captures and destroys pathogens, and induces adaptive immune responses [3]. Therefore, the clarification of spleen regulatory mechanisms could help us further understand the immune system of the giant panda as well as its conservation.

RNA-seq is a powerful method for analyzing global gene expression levels and providing in-depth analysis of the transcriptome. Since the giant panda genome had already been reported [4], previous studies of the giant panda transcriptome have mainly focused on mRNA levels. Thus far, transcriptional profiling of mRNA expression in blood and 12 types of giant panda tissues have been determined $[5,6]$. However, the spleen has not yet been investigated.

In addition to mRNA, non-coding RNAs (ncRNAs) are another class of RNA molecules. Among the 3 billion base pairs of the human genome, only $2 \%$ encode proteins. Indeed, except for about $7 \%$ of the non-transcribed regions, the remaining $91 \%$ of the genome encodes noncoding RNAs (The ENCODE Project Consortium, Nature, 2007). Among ncRNAs, microRNAs (miRNAs) have been particularly well characterized, and they can act as small regulatory RNA molecules for mRNA expression [7, 8]. Although a study on miRNAs in giant panda blood was previously reported [9], miRNAs in giant panda spleens, important organs in the immune system as described above, have not been investigated yet.

Moreover, another major class of ncRNAs is long non-coding RNAs (lncRNAs), which have lengths exceeding 200 nucleotides. Some lncRNAs have been found to be associated with pathogenesis of a number of diseases [10]. Furthermore, circular RNAs (circRNAs), newly discovered endogenous non-coding RNAs, have been demonstrated to play important roles in a wide range of biological processes [11-15]. However, no study of lncRNAs and circRNAs in giant panda have been reported.

In view of the importance of the spleen in the immune system and based upon the current progress of ncRNA research in giant panda, in this study, we determined the whole transcriptional profile of two giant panda spleens, one from a newborn and another from an adult. Since newborn immune cells are qualitatively distinct from adult cells [16], the study may reveal the regulatory mechanisms of spleen immune responses by ncRNAs.

\section{Materials and Methods}

\section{Ethics statement}

Samples collection and utility protocols were approved by the Chengdu Institute of Biology Animal Use Ethics, which is responsible for the Chengdu Research Base of Giant Panda Breeding.

\section{Sample preparation, transcriptome library construction, and sequencing}

The spleen samples were collected from two recently deceased giant pandas and immediately stored in liquid nitrogen until RNA extraction. The two giant pandas were both male. One was a newborn individual that died by asphyxiation 4 days after birth, and the other is an adult individual that died by accident.

A total amount of $1.5 \mu \mathrm{g}$ of RNA per sample was used as input material for rRNA removal using the Ribo-Zero rRNA Removal Kit (Epicentre, Madison, WI). RNA depleted libraries were generated with NEB Next $^{\mathrm{R}}$ Ultra $^{\mathrm{TM}}$ Directional RNA Library Prep Kit for Illumina R (New England BioLabs, Inc., Ipswich, MA) in 
accordance with the manufacturer's recommendations while miRNA sequencing libraries were generated with the NEB Next Ultra small RNA Sample Library Prep Kit for Illumina (New England BioLabs, Inc.) according to the manufacturer's instructions. Library quality was assessed on the Agilent Bioanalyzer 2100 (Agilent Technologies, Santa Clara, CA) and qPCR. The libraries were sequenced by using Illumina HiSeq platform (Illumina, San Diego, CA) and paired-end reads or single-end reads were generated.

\section{Data processing of RNA depleted libraries}

Raw data (raw reads) in fastq format were first processed using in-house Perl scripts. In this step, clean data (clean reads) were obtained by removing reads containing adapters, reads containing poly- $\mathrm{N}$ sequences, and low-quality reads from the raw data. Secondly, Q20, Q30, GC-content, and sequence duplication level of the clean data were inferred. All the downstream analyses were based on high-quality clean data.

The transcriptome was assembled using Cufflinks and Scripture analyses based on the reads mapped to the reference giant panda genome. Sequences with the smallest number of Ns and that could not be extended in either direction were defined as unigenes. The assembled transcripts were annotated using the Cuffcompare program from the Cufflinks package. The unknown transcripts were used to screen for putative lncRNAs. Three computational approaches (CPC, CNCI, and Pfam) were combined to sort nonprotein coding RNA candidates from putative protein-coding RNAs among the unknown transcripts. Putative protein-coding RNAs were filtered out using a minimum length and exon number threshold. Transcripts of more than $200 \mathrm{nt}$ and more than two exons were selected as lncRNA candidates and further screened using $\mathrm{CPC}$, and $\mathrm{CNCI}$, and Pfam, which are collectively able to distinguish protein-coding genes from non-coding genes. The different types of lncRNAs, including lincRNAs, intronic lncRNAs, and anti-sense lncRNAs, were selected using Cuffcompare.

\section{Identification of circular RNAs}

CIRI software [17] was used to identify circular RNAs. First, CIRI employed BWA to align the clean reads with a reference genome to generate SAM files. Then, CIRI was used to analyze the CIGAR values and detect junction reads with paired chiastic clipping (PCC) signals. Second, CIRI scanned SAM files to detect additional junction reads and eliminate false positive candidates. Differentially expressed circRNAs were identified using EBSeq.

Prediction of targets of IncRNAs and circRNAs and construction of IncRNA-miRNA-mRNA association networks

The lncRNAs targets were identified using two strategies: cis and trans regulation prediction. For the cis prediction, we first identified the genomic location of the paired IncRNAs and mRNAs, and genes within $100 \mathrm{~kb}$ of IncRNAs were regarded as potential targets. For trans prediction, we screened the targets by comparing complementary bases between IncRNAs and mRNAs. LncTar [18] was used to calculate the free energy between IncRNAs and mRNAs and to predict the targets of lncRNAs.

The IncRNA-miRNA and miRNA-mRNA interactions were predicted by miRcode and TarBase, respectively. Potential miRNA targets of circRNAs were based on miRanda and TargetScan.

\section{Quantification of gene expression levels}

Cuffdiff (v2.1.1) was used to calculate FPKMs of both lncRNAs and coding genes in each sample. Gene fragments per kilobase of exon per million fragments mapped (FPKMs) were computed by summing the FPKMs of transcripts in each gene group. FPKM was calculated based on the length of the fragments and reads mapped to each fragment.

\section{Differential expression analysis and gene functional annotation}

Differential expression analysis of the two samples was performed using the EBseq (2010) R package. $P$-values were based on adjusted $q$-values. $Q<0.01 \& \mid \log 2$ (fold change) $\mid>1$ was used as the threshold for determining significantly differential expression.

Gene function was annotated based on the following databases: NCBI non-redundant protein sequences (Nr), Protein Family (Pfam), Clusters of Orthologous Groups (COG)/euKaryotic Orthologous Groups (KOG), Swiss-Prot (a manually annotated and reviewed protein sequence database), Kyoto Encyclopedia of Genes and Genomes (KEGG), and Gene Ontology (GO).

\section{KARGER}




\section{Cellular Physiology Cell Physiol Biochem 2018;46:1065-1077 \begin{tabular}{l|l} 
and Biochemistry Published $10.1159 / 000488837$ & $\begin{array}{l}\text { DO } 2018 \text { The Author(s). Published by S. Karger AG, Basel } \\
\text { www.karger.com/cpb }\end{array}$
\end{tabular}}

Peng et al.: Characters of ncRNAs in Giant Panda Spleen

GO enrichment analysis of the differentially expressed genes (DEGs) was implemented using the topGO R packages. KOBAS software [19] was used to test the statistical enrichment of DEGs in KEGG pathways.

Data processing of miRNA libraries

Bowtie tools software was used to map the clean reads with the Silva database, GtRNAdb database, Rfam database, and Repbase database sequence alignments, respectively, and then, ribosomal RNA (rRNA), transfer RNA (tRNA), small nuclear RNA (snRNA), small nucleolar RNA (snoRNA), and other ncRNA and repeats were filtered. The remaining reads were used to detect known miRNA and new miRNA that was predicted by comparing the data with known miRNAs from miRBase. Randfold tools soft was used for new miRNA secondary structure prediction. Target gene function was annotated based on the following databases: Nr, Nt, Pfam, KOG/COG, Swiss-Prot, KEGG, and GO.

\section{Quantification of miRNA expression levels}

miRNA expression levels were estimated for each sample. First, sRNAs were mapped back onto the precursor sequence. Then, read count for each miRNA was obtained from the mapping results.

\section{Differential expression analysis}

For each sequenced library, differential expression analysis of the two samples was performed using IDEG6 [20]. The $P$-value was adjusted using the $q$-value. $Q<0.005 \& \log 2$ (fold change) $\geq 1$ was used as the threshold for determining significantly differential expression.

\section{Results}

\section{RNA-seq of spleen transcriptome}

To better understand the molecular changes in the immune response of the adult giant panda, we sequenced the whole transcriptome, including mRNA, IncRNA, miRNA, and circRNA. Two kinds of cDNA libraries, an rRNA depleted library and an miRNA library, were constructed from the spleens of the newborn and adult giant pandas. High-throughput paired-end sequencing of the rRNA depleted library yielded 79, 403, 439 and 61, 299, 854 total clean reads from the spleens of the newborn and adult giant pandas, respectively, which were used to identify the mRNAs, IncRNAs, and circRNAs. Paired-end sequencing of the miRNA library yielded 36, 575, 946 and 52, 045, 245 total clean reads from the spleens of the newborn and adult giant pandas, respectively. The raw reads are available from the NCBI SRA browser (Bioproject accession number, PRJNA396970).

Transcriptional profiling of mRNA expression in giant panda spleens at different developmental stages

In addition to the unigenes annotated by Li et al [4]., 1, 549 new unigenes were identified in this study. All of the new annotated unigenes were classified into three main categories in the GO database, including cellular component, molecular function, and biological process (Fig. 1A). To further investigate the gene expression pattern of these two differently aged giant panda spleens, DEGs were identified using FPKM. With the criteria of fold change $\geq 2$ and false detection rate $<0.05$, a total of 3,466 differentially expressed genes were identified, of which 1, 992 showed up-regulation and 1, 474 showed down-regulation in the adult compared with the newborn individual.

To generate the functional distribution of the differentially expressed genes, a GO analysis of these genes was performed. Finally, 2, 369 genes were classified into three major functional categories. Among them, the largest numbers of DEGs were clustered in biological process, followed by cellular component and molecular function. Additionally, we conducted a KEGG analysis to generate overviews of the functions and interactions of these DEGs. KEGG analysis showed that a total of 1, 026 genes were assigned to 261 pathways corresponding to five categories, including Environmental Information Processing (531), Human Diseases (566), Organismal Systems (323), Cellular Processes (171), and Metabolism (60) (Fig. 1B). 


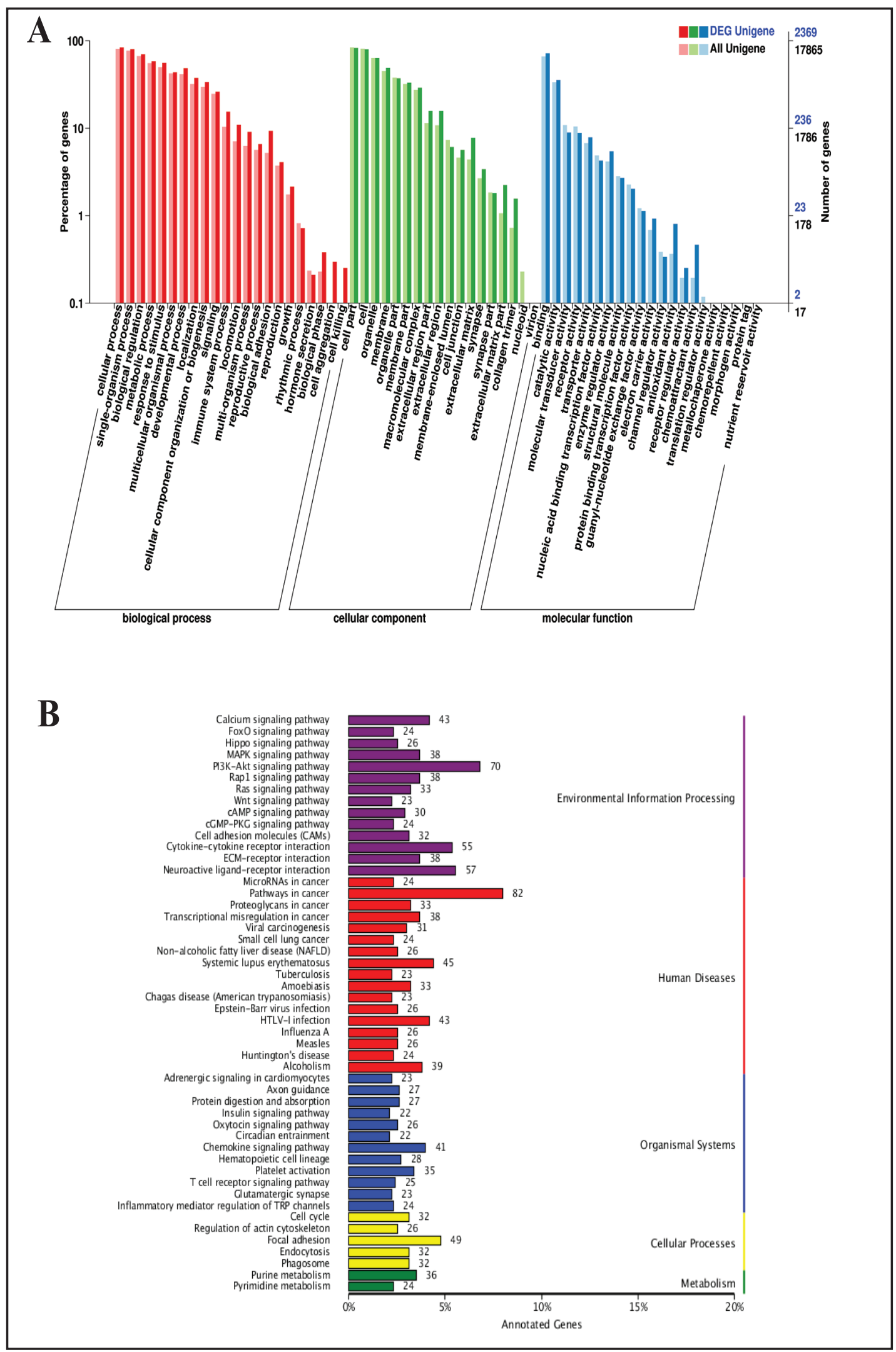

Fig. 1. Gene ontology (GO) and KEGG assignments for the differentially expressed mRNAs between the newborn and adult giant panda spleens. (A) GO assignment; (B) KEGG assignment. 
Among these pathways, 50 showed statistically significant overrepresentation $(P<0.05$; Table 1), and the most significant pathway was systemic lupus erythematosus, in which some immune-relevant genes were significantly up-regulated, such as MHCII, CD28, CD80, TRIM62, FAS, IL12RB1, IL34, and CCL19. Among these 50 significant pathways, 9 pathways are related to immunity. The 50 significant pathways are summarized in Table 1.

MicroRNAs regulate a variety of processes including immune responses in giant panda spleen

A total of 737 microRNAs were identified from the two giant panda spleens. The TPM density and correlation coefficient showed that the miRNA expression levels were highly consistent between the newborn and adult spleens (Fig. 2A, B). Furthermore, we found a total of 503 differentially expressed microRNAs. Interestingly, most of the differentially expressed microRNAs (408 out of 503) were highly expressed in the newborn spleen, when compared to the adult spleen in which only 95 microRNAs were up-regulated.

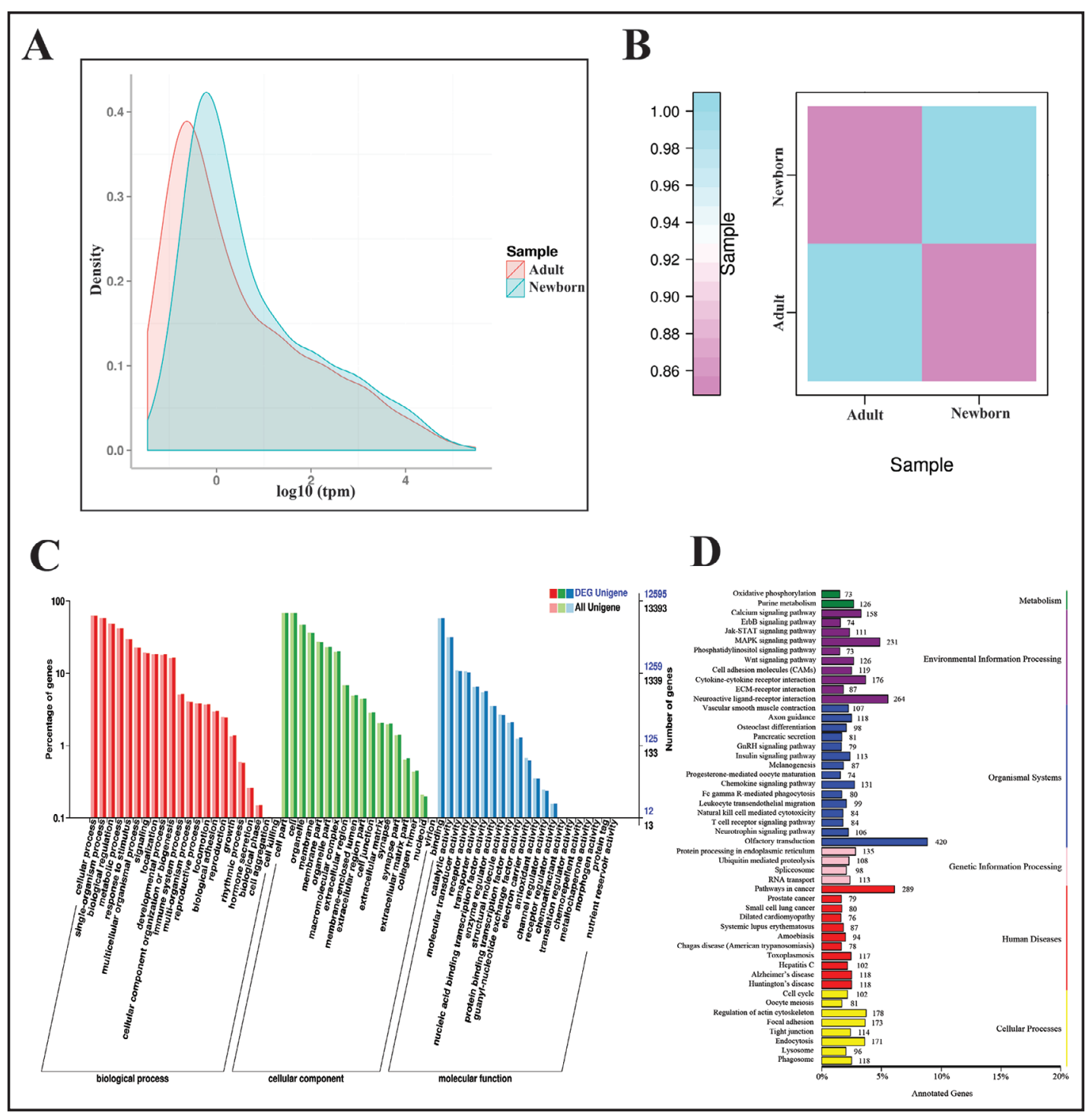

Fig. 2. microRNA characteristics of giant panda spleen. (A) TPM density of microRNA expression between newborn and adult giant panda spleens; (B) Correlation coefficient of microRNA expression between newborn and adult giant panda spleens; GO assignment (C) and KEGG assignment (D) of differentially expressed microRNAs between the newborn and adult giant panda spleens. 
To further investigate the functions of these differentially expressed microRNAs, their target genes were predicted. Similar to the mRNA expression results, the largest numbers of target genes were clustered in biological processes, followed by cellular component and molecular function (Fig. 2C). KEGG analysis revealed that 4,750 target genes were mapped to six categories, and one additional category, Genetic Information Processing, was included when comparing them to DEGs (Fig. 2D). In addition, 11 pathways were statistically significant, and only one pathway, the $\mathrm{T}$ cell receptor signaling pathway, was associated with immunity (Table 2).

One of the major functions of IncRNAs is to regulate immune response

It has been reported that lncRNAs play important roles in various biological processes, such as transcription, post-transcription, and epigenetic regulation. We used Coding Potential Calculator (CPC), CodingNon-Coding Index (CNCI), Coding Potential Assessment Tool (CPAT), and Pfam to predict the coding potential of candidate non-coding transcripts. The intersection of $\mathrm{CPC}, \mathrm{CNCI}, \mathrm{CPAT}$, and Pfam revealed 5, 992 non-coding transcripts, and 2, 987 putative IncRNAs were ultimately identified, which is the first reported IncRNA estimate for giant pandas. Furthermore, the DEG analysis showed that 239 lncRNAs were up-regulated and 173 were down-regulated in the adult individual relative to the newborn.

To investigate the characteristics of giant panda IncRNAs, we then compared the length, exon number, and expression levels of lncRNAs to protein-coding genes. As shown in Fig. 3, lncRNAs were shorter (2, 045 versus 2, 620 nucleotides) with fewer exons per transcript
Table 1. Statistically significant immune-related KEGG classifications of differentially expressed genes in giant panda spleens

\begin{tabular}{lccc}
\hline KEGG pathway & Pathway ID & Gene No. (\%) & P-value \\
\hline Systemic lupus erythematosus & ko05322 & $4.39 \%$ & $4.72 \mathrm{E}-12$ \\
Hematopoietic cell lineage & ko04640 & $2.73 \%$ & $2.46 \mathrm{E}-05$ \\
Platelet activation & ko04611 & $3.41 \%$ & 0.000211247 \\
Chemokine signaling pathway & ko04062 & $4.00 \%$ & 0.00159361 \\
Primary immunodeficiency & ko05340 & $1.27 \%$ & 0.002320047 \\
Intestinal immune network for IgA production & ko04672 & $1.46 \%$ & 0.013117497 \\
Complement and coagulation cascades & ko04610 & $1.75 \%$ & 0.013933835 \\
T cell receptor signaling pathway & ko04660 & $2.44 \%$ & 0.015570937 \\
Rheumatoid arthritis & ko05323 & $1.95 \%$ & 0.045095082 \\
\hline
\end{tabular}

Table 2. Statistically significant KEGG classifications of the genes targeted by differentially expressed miRNAs in giant panda spleens. * indicated immune-related pathway

\begin{tabular}{lccc}
\hline KEGG pathway & Pathway ID & Gene No. (\%) & P-value \\
\hline ECM-receptor interaction & ko04512 & $1.83 \%$ & 0.003890846 \\
Axon guidance & ko04360 & $2.48 \%$ & 0.004417373 \\
T cell receptor signaling pathway* & ko04660 & $1.77 \%$ & 0.0047192 \\
TGF-beta signaling pathway & ko04350 & $1.49 \%$ & 0.010876896 \\
VEGF signaling pathway & ko04370 & $1.39 \%$ & 0.014987211 \\
Toxoplasmosis & ko05145 & $2.46 \%$ & 0.019860763 \\
Basal cell carcinoma & ko05217 & $1.12 \%$ & 0.034435747 \\
Small cell lung cancer & ko05222 & $1.68 \%$ & 0.036496257 \\
Cell cycle & ko04110 & $2.15 \%$ & 0.041208505 \\
Acute myeloid leukemia & ko05221 & $1.05 \%$ & 0.041710585 \\
Osteoclast differentiation & ko04380 & $2.06 \%$ & 0.049796733 \\
\hline
\end{tabular}

Table 3. Statistically significant KEGG classifications of the genes targeted by differentially expressed lncRNAs in giant panda spleens. * indicated immune-related pathway

\begin{tabular}{lccc}
\hline KEGG pathway & Pathway ID & Gene No. (\%) & P-value \\
\hline Toll-like receptor signaling pathway* & ko04620 & $6.34 \%$ & 0.000116843 \\
RIG-I-like receptor signaling pathway* & ko04622 & $4.23 \%$ & 0.001288695 \\
Fc epsilon RI signaling pathway* & ko04664 & $4.23 \%$ & 0.002266396 \\
T cell receptor signaling pathway* & ko04660 & $4.93 \%$ & 0.006946106 \\
Platelet activation* & ko04611 & $4.93 \%$ & 0.016908702 \\
Fc gamma R-mediated phagocytosis* & ko04666 & $3.52 \%$ & 0.033709797 \\
Natural killer cell mediated cytotoxicity* & ko04650 & $3.52 \%$ & 0.047682419 \\
\hline
\end{tabular}




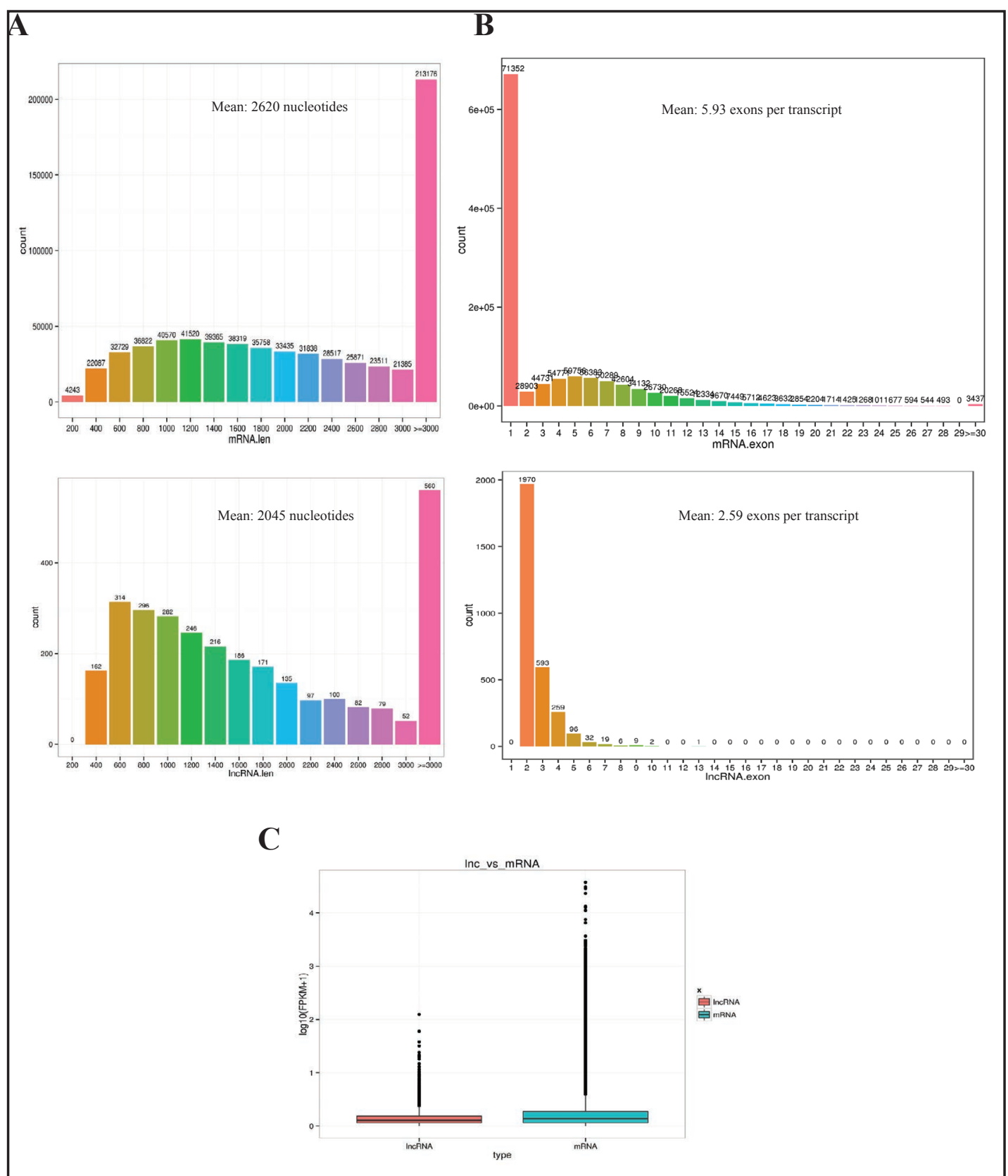

Fig. 3. IncRNA characteristics of giant panda spleen. (A) Distribution of transcript lengths of mRNAs (upper) and lncRNAs (lower); (B) Distribution of exon numbers of mRNAs (upper) and lncRNAs (lower); (C) Comparison of the expression levels of mRNAs and lncRNAs.

(about 2.59 versus 5.93) relative to protein-coding genes. In addition, expression levels of lncRNAs were slightly lower than those of protein-coding genes (Fig. $3 \mathrm{C}$ ).

To determine the functions of differentially expressed lncRNAs, we employed two independentalgorithms, cis (genomic location) and trans (mRNA sequence complementarity), to predict the target genes of all newly identified lncRNAs. Finally, target genes of 2, 258 lncRNAs were predicted. Among these, 391 target genes of differentially expressed lncRNAs were found. KEGG analysis revealed that the target genes of differentially expressed lncRNAs were assigned to 197 pathways within six categories, and 34 pathways showed statistically significant differential expression $(P<0.05$; Table 3$)$. Of these 34 pathways, 7 were related 
to immune responses, and we found that 10 differentially expressed lncRNAs may regulate 6 differentially expressed immune-related genes via trans effects (Table 4), implying that IncRNAs may be involved in the regulation of spleen immune responses.

To further investigate the role of IncRNAs in immune responses, we next constructed IncRNA-miRNA-mRNA association networks including lncRNAs and immune-related target genes. The network results indicated connections between 67 lncRNAs and 46 immunerelated mRNAs. Of these 67 lncRNAs, 11 showed differential expression between newborn and adult spleens and may regulate 10 important immune-related genes via microRNAs (Table 4), implying a major role of lncRNAs in the regulation of immune responses.

\section{Expression patterns of circRNAs}

Using CIRI, we identified a total of 9, 497 and 9, 609 circRNAs in newborn and adult giant panda spleens, respectively, and 7, 628 circular RNAs were shared (Fig. 4A). Finally, a total of 11, 478 circRNAs were identified in giant panda spleens. Among the identified circRNAs, $8,088(70.5 \%)$ of the circRNAs were generated from exons of protein-coding genes, 2,769 $(24.1 \%)$ were generated from intergenic regions, and the remaining $(621,5.4 \%)$ were from introns (Fig. 4B), suggesting that circRNAs in giant panda spleens were from diverse genomic regions.

A total of 41 circRNAs showed differential expression between newborn and adult giant panda spleens. The length of the 41 differentially expressed circRNAs ranged from 148 nt to $41,333 \mathrm{nt}$, with an average length of about 5, 000 nt. It has been reported that circRNAs can bind to miRNAs and consequently repress their function. To detect whether circRNAs could affect gene post-transcriptional regulation by binding to miRNAs, we predicted target miRNAs of all 41 differentially expressed circRNAs. Surprisingly, these 41 differentially expressed circRNAs could bind to 728 (98.8\%) of all identified miRNAs, suggesting that circRNAs may play important roles as miRNA sponges across a wide array of biological processes in the spleen.

\section{Discussion}

Giant pandas can suffer from a variety of diseases. Due to their highly specialized diet of bamboo, the giant panda was thought

Table 4. The differentially expressed immune genes regulated by differentially expressed IncRNAs

\begin{tabular}{llcc}
\hline \multicolumn{2}{c}{ IncRNA } & Targeted gene name & $\begin{array}{c}\text { Targeted } \\
\text { gene ID }\end{array}$ \\
\hline $\begin{array}{c}\text { trans } \\
\text { effect }\end{array}$ & TCONS_00171778 & LBP & gene4086 \\
& TCONS_00750761 & IgG & gene28094 \\
& TCONS_00611513;TCONS_00611514;TCONS_00611663;TC & SLP-76 & gene15141 \\
& ONS_00611512;TCONS_06611664 & GPIB $\alpha$ & gene24361 \\
& TCONS_00729282 & SPHK & gene10906 \\
& TCONS_00446647 & FAS & gene2957 \\
& TCONS_00122418 & FAS & gene2957 \\
Cis & TCONS_00122418 & & gene4086, \\
effect & & LOC100476795, & gene4088 \\
& TCONS_00171778 & LBP & gene4648 \\
& TCONS_00197620; TCONS_00197807 & LIF & gene9850, \\
& TCONS_00405916; TCONS_00406016 & LOC105240615, & gene9851 \\
& TCONS_00497823; TCONS_00497835 & LOC100484936 & gene12072 \\
& TCON_00532954 & WNT7A & gene13060 \\
& TCONS_00561530 & ICAM5 & gene13746 \\
& TCONS_00750761 & LCK & gene28094 \\
\hline
\end{tabular}

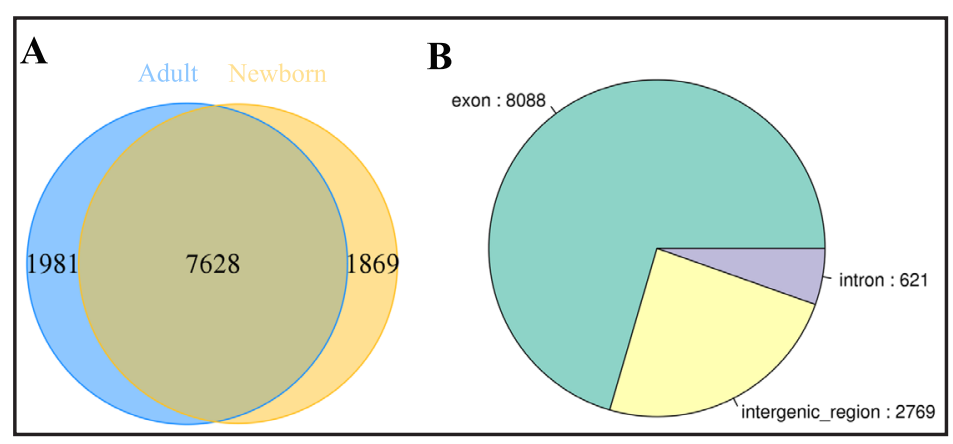

Fig. 4. The number of newly identified circRNAs in newborn and adult giant panda spleens (A) and the source and distribution of these circRNAs (B). 
to have a relatively weak resistance to diseases [21]. However, a previous study reported that one of the important immune-related genes, the major histocompatibility complex (MHC), showed high genetic diversity in wild giant panda populations [22]. This may suggest that the giant panda immune system is stronger than previously thought. Nevertheless, the main problem for giant panda captive breeding is still infectious diseases. Thus, improving their immunity and enhancing disease resistance is a critical issue in giant panda breeding as well as conservation.

Previous studies have shown that ncRNAs can regulate gene expression at the transcriptional, post-transcriptional, and epigenetic levels [23-25]. Until now, only one study had identified microRNAs from the blood of giant pandas [9]. To further investigate the regulatory mechanism of immune responses in giant panda spleen, we analyzed the whole transcriptomes of two male individuals' spleens; one was a newborn, and the other was an adult. Comparing the whole transcriptomes of these two spleens can provide insight into the regulatory mechanisms of immune response at all transcriptomic levels, including among mRNAs, microRNAs, IncRNAs, and circRNAs.

A total of 3, 466 DEGs were identified between the spleens of newborn and adult giant pandas. KEGG analysis reveals Systemic lupus erythematosus as the most significant pathway, in which some important immune-relevant genes, such as MHCII, CD28, CD80, TRIM62, FAS, IL12RB1, IL34, and CCL19, were significantly up-regulated. MHCII molecules are normally found only on antigen-presenting cells and play important roles in the adaptive immune systems of vertebrates against intracellular pathogens [26]. CD28 and CD80 are both crucial immune genes that play important roles in protective immune responses against foreign pathogens and exacerbation of lymphoproliferation and autoimmunity [27, 28]. Deficiencies of TRIM62 and IL12RB1 showed an increased susceptibility to fungal infection in mouse and immunodeficiency of patients with severe mycobacterial and Salmonella infections respectively [29, 30]. Like IL12RB1, IL34 is an interleukin protein. IL34 expression is most abundant in spleen and found to be involved into growth and development of myeloid cells [31]. CCL19, one of several CC cytokine genes, also plays an important role in attracting certain cells of the immune system and is involved in immune regulatory and inflammatory processes [32-34]. In addition, our results also showed that DEGs were not only associated with immune system pathways but also with other pathways, for example, the ECM-receptor interaction and cytokine-cytokine receptor interaction. This result may be a consequence of the different developmental stages of these two samples.

In this study, we identified a total of 737 microRNAs from two giant panda spleens, far more than that reported by a previous study (327 microRNAs) [6]. The previous study showed that target genes of microRNAs from blood were mainly involved in host immunity, including the Ras signaling pathway, the PI3K-Akt signaling pathway, and the MAPK signaling pathway [6]. However, we found that most target genes of the differentially expressed miRNAs were involved in the CM-receptor interaction pathway, following the Axon guidance and $\mathrm{T}$ cell receptor signaling pathways. Among all 11 statistically significant pathways, only one pathway was involved in immunity. Interestingly, we found that most miRNAs were highly expressed in the newborn spleen but down-regulated in the adult spleen. Considering the function roles of miRNAs, we suspected that microRNAs may be involved in the regulation of not only immune responses but also other biological processes.

LncRNA is another major class of ncRNAs and could act as important regulators of gene expression in a wide variety of biological processes, including immune responses [35, 36]. It has been reported that lincRNA-Cox 2 could be induced in mouse macrophages after activation of Toll-like receptors so as to detect microbes and alert the immune system to respond [37]. In this study, we first identified 2, 987 putative lncRNAs of giant panda. A total of 412 differentially expressed miRNAs were found between the newborn and adult spleen. It has been shown that lncRNAs regulate transcription via trans and cis effects in many different ways [37]. The prediction of lncRNA target genes would be useful for identifying which processes lncRNAs participated in as well as their potential functions [38]. We predicted the target genes of differentially expressed lncRNAs and conducted a KEGG

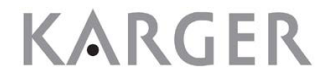


analysis. The results revealed 7 of these 34 statistically significant pathways were related to immune responses. Furthermore, our results showed that 19 differentially expressed lncRNAs may regulate 14 important immune-related genes via trans and cis effects, such as LBP (lipopolysaccharide binding protein), FAS, LIF, IgG, GPIB $\alpha$, SPHK, WNT7A, ICAM5, and LCK. In addition, LBP and FAS were shown to be regulated by lncRNAs via both trans and cis effects. LBP is involved in the acute-phase immunologic response to gram-negative bacterial infections. LBP could bind to lipopolysaccharide-aggregates or bacteria and facilitate the extraction of lipopolysaccharide monomers by the extra-cellular protein CD14, playing a role in regulating lipopolysaccharide-dependent monocyte responses [39]. FAS is a member of the TNF-receptor superfamily and plays critical roles in the immune system, including the killing of pathogen-infected cells and the death of obsolete and potentially dangerous lymphocytes [40]. Together, our results revealed that one of the main roles of IncRNAs is to regulate spleen immune responses in giant panda.

In addition to mRNAs, microRNAs, and IncRNAs, circRNAs are a distinct class of newly discovered endogenous ncRNAs. Indeed, circRNAs were found to play important roles in various biological and developmental processes across a wide range of organisms [11-15]. There is little known about the features of circRNAs in giant panda. To explore the expression patterns of circRNAs and their potential function in the regulation of immune responses, we first identified circRNAs in giant pandas. Finally, we found that a total of 11, 478 circRNAs and 41 circRNAs showed differential expression between newborn and adult giant panda spleens. An analysis of circRNA-miRNA associations showed that 41 differentially expressed circRNAs can regulate $98.8 \%$ of all identified miRNAs in giant panda spleens, suggesting circRNAs may also play important roles in the regulation of a wide range of biological processes, not limited to immune responses.

Taken together, this study has investigated whole transcriptome profiles of two giant panda spleens from newborn and adult individuals. We have identified 737 microRNAs, 2, 987 putative lncRNAs, and 11, 478 circRNAs in giant panda spleens. To our knowledge, this is the first effort to explore the profiles of IncRNAs and circRNAs in giant pandas. Moreover, a combination analysis showed that microRNAs and circRNAs can regulate a wide range of biological processes in giant panda spleens. More interestingly, the present study has established that lncRNAs may play an important role in the regulation of immune responses in giant panda spleens.

Giant panda is a powerful symbol of species conservation. Our study shed light on the function of lncRNAs in immune responses and protection from diseases, which may benefit the future conservation of giant pandas. Further functional study may be needed to clarify the detailed regulatory mechanisms of lncRNAs in giant panda immune responses.

\section{Acknowledgements}

We thank Dr. Shang-mian Yie at Chengdu Research Base of Giant Panda Breeding for his helpful comments on the manuscript.

\section{Disclosure Statement}

No conflict of interests exists.

\section{References}

1 Zou X, Sun Z, Yan W, He G, Wu K, Chen Y, Weng N: Analysis of Lethal Factors of Diseased Giant Pandas and Prevention Countermeasures. J Northeast Forestry University 1998;26:53-56. 


\section{Cellular Physiology Cell Physiol Biochem 2018;46:1065-1077

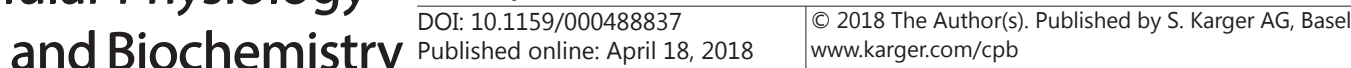

Peng et al.: Characters of ncRNAs in Giant Panda Spleen

2 Zhao N, Li M, Luo J, Wang S, Liu S, Wang S, He HX: Impacts of canine distemper virus infection on the giant panda population from the perspective of gut microbiota. Sci Rep 2017;7:39954.

-3 Mebius RE, Kraal G: Structure and function of the spleen. Nature Rev Immunol 2005;5:606-616.

4 Li R, Fan W, Tian G, Zhu H, He L, Cai J, Wang J: The sequence and de novo assembly of the giant panda genome. Nature 2010;463:311-317.

-5 Chen ML, Hu YB, Liu JX, Wu Q Zhang CL, Yu J,Xiao JF, Wei FW, Wu JY: Improvement of genome assembly completeness and identification of novel full-length protein-coding genes by RNA-seq in the giant panda genome. Sci Reports 2015;5:18019.

-6 Du L, Li W, Fan Z, Shen F, Yang M, Wang Z,Jian Z, Hou R, Yue B, Zhang X: First insights into the giant panda (Ailuropoda melanoleuca) blood transcriptome: a resource for novel gene loci and immunogenetics. Mol Ecol Resour 2015;15:1001-1013.

7 Janga SC, Vallabhaneni S: MicroRNAs as post-transcriptional machines and their interplay with cellular networks. Adv Exp Med Biol 2011;722:59-74.

-8 Zhao Y, He SM, Liu CN, Ru SW, Zha HT, Yang Z,Yang P, Yuan x, Sun S, Bu D, Huang J, Skogerbø G, Chen R: MicroRNA regulation of messenger-like noncoding RNAs: a network of mutual microRNA control. Trends Genet 2008;24:323-327.

9 Yang M, Du L, Li W, Shen F, Fan Z, Jian Z,Hou R, Shen Y, Yue B, Zhang X: Profile of microRNA in Giant Panda Blood: A Resource for Immune-Related and Novel microRNAs. PLoS One 2015;10:e0143242.

10 Bhartiya D, Kapoor S, Jalali S, Sati S, Kaushik K, Sachidanandan C, Scaria V: Conceptual approaches for lncRNA drug discovery and future strategies. Expert Opin Drug Dis 2012;7:503-513.

11 Danan M, Schwartz S, Edelheit S, Sorek R: Transcriptome-wide discovery of circular RNAs in Archaea. Nucleic Acids Res 2012;40: 3131-3142.

12 Guo JU, Agarwal V, Guo HL, Bartel DP: Expanded identification and characterization of mammalian circular RNAs. Genome Biol 2014;15:409.

-13 Jeck WR, Sharpless NE: Detecting and characterizing circular RNAs. Nature Biotechnol 2014;32:453-461.

-14 Salzman J, Chen RE, Olsen MN, Wang PL, Brown PO: Cell-Type Specific Features of Circular RNA Expression. Plos Genet 2013:9:e1003777.

15 Zhang Y, Zhang XO, Chen T, Xiang JF, Yin QF, Xing YH,Zhu S, Yang L, Chen LL: Circular Intronic Long Noncoding RNAs. Mol Cell 2013;51:792-806.

16 Adkins B, Leclerc C, Marshall-Clarke S: Neonatal adaptive immunity comes of age. Nat Rev Immunol 2004;4:553-564.

17 Gao Y, Wang J, Zhao F: CIRI: an efficient and unbiased algorithm for de novo circular RNA identification. Genome Biol 2015;16:4.

18 Li J, Ma W, Zeng P, Wang J, Geng B, Yang J, Cui Q: LncTar: a tool for predicting the RNA targets of long noncoding RNAs. Brief Bioinform 2015;16:806-812.

19 Mao XZ, Cai T, Olyarchuk JG, Wei LP: Automated genome annotation and pathway identification using the KEGG Orthology (KO) as a controlled vocabulary. Bioinformatics 2005;21: 3787-3793.

20 Romualdi C, Bortoluzzi S, D’Alessi F, Danieli GA: IDEG6: a web tool for detection of differentially expressed genes in multiple tag sampling experiments. Physiol Genomics 2003;12:159-162.

-21 Peng R, Zeng B, Meng X, Yue B, Zhang Z, Zou F: The complete mitochondrial genome and phylogenetic analysis of the giant panda (Ailuropoda melanoleuca). Gene 2007;397:76-83.

22 Pan HJ, Wan QH, Fang SG: Molecular characterization of major histocompatibility complex class I genes from the giant panda (Ailuropoda melanoleuca). Immunogenetics 2008;60:185-193.

23 Hangauer MJ, Vaughn IW, McManus MT: Pervasive Transcription of the Human Genome Produces Thousands of Previously Unidentified Long Intergenic Noncoding RNAs. Plos Genet 2013;9:e1003569.

-24 Kim ED, Sung S: Long noncoding RNA: unveiling hidden layer of gene regulatory networks Trends. Plant Sci 2012;17:16-21.

25 Wapinski O, Chang HY: Long noncoding RNAs and human disease. Trends Cell Biol 2011;21 354-361.

26 Walseng E, Furuta K, Bosch B, Weih KA, Matsuki Y, Bakke O, Ishido S, Roche PA: Ubiquitination regulates MHC class II-peptide complex retention and degradation in dendritic cells. Proc Natl Acad Sci USA 2010;107:20465-20470.

27 Bour-Jordan H, Esensten JH, Martinez-Llordella M, Penaranda C, Stumpf M, Bluestone JA: Intrinsic and extrinsic control of peripheral T-cell tolerance by costimulatory molecules of the CD28/B7 family. Immunol Rev 2011;241:180-205. 


\section{Cellular Physiology Cell Physiol Biochem 2018;46:1065-1077 \begin{tabular}{l|l} 
DOI: 10.1159/000488837 & $\begin{array}{l}\text { O 2018 The Author(s). Published by S. Karger AG, Basel } \\
\text { wwww.karger.com/cpb }\end{array}$
\end{tabular}}

Peng et al.: Characters of ncRNAs in Giant Panda Spleen

28 Weintraub JP, Cohen PL: Ectopic expression of B7-1 (CD80) on T lymphocytes in autoimmune lpr and gld mice. Clin Immunol 1999;91:302-309.

-29 Cao ZF, Conway KL, Heath RJ, Rush JS, Leshchiner ES, Ramirez-Ortiz ZG,Nedelsky NB, Huang H, Ng A, Gardet A, Cheng SC, Shamji AF, Rioux JD, Wijmenga C, Netea MG, Means TK, Daly MJ, Xavier RJ: Ubiquitin Ligase TRIM62 Regulates CARD9-Mediated Anti-fungal Immunity and Intestinal Inflammation.Immunity 2015;43:715-726.

-30 de Jong R, Altare F, Haagen IA, Elferink DG, de Boer T, van Breda Vriesman PJ, Kabel PJ, Draaisma JM, van Dissel JT, Kroon FP, Casanova JL, Ottenhoff TH: Severe mycobacterial and Salmonella infections in interleukin-12 receptor-deficient patients. Science 1998;280:1435-1438.

31 Lin HS, Lee E, Hestir K, Leo C, Huang MM, Bosch E, Williams LT: Discovery of a cytokine and its receptor by functional screening of the extracellular proteome. Science 2008;320:807-811.

32 Reif K, Ekland EH, Ohl L, Nakano H, Lipp M, Forster R, Cyster JG: Balanced responsiveness to chemoattractants from adjacent zones determines B-cell position. Nature 2002;416:94-99.

33 Robbiani DF, Finch RA, Jager D, Muller WA, Sartorelli AC, Randolph GJ: The leukotriene C-4 transporter MRP1 regulates CCL19 (MIP-3 beta ELC)-dependent mobilization of dendritic cells to lymph nodes. Cell 2000;103:757-768.

34 Yoshida R, Imai T, Hieshima K, Kusuda J, Baba M, Kitaura M,Nishimura M, Kakizaki M, Nomiyama H, Yoshie O: Molecular cloning of a novel human CC chemokine EBI1-ligand chemokine that is a specific functional ligand for EBI1 CCR7 Journal of Biological Chemistry 1997;272:13803-13809.

35 Carpenter S, Aiello D, Atianand MK, Ricci EP, Gandhi P, Hall LL,Byron M, Monks B, Henry-Bezy M, Lawrence JB, O’Neill LA, Moore MJ, Caffrey DR, Fitzgerald KA: A Long Noncoding RNA Mediates Both Activation and Repression of Immune Response Genes. Science 2013;341:789-792.

-36 Carpenter S, Atianand M, Aiello D, Ricci E, Gandhi P, Hall LL,Byron M, Monks B, Henry-Bezy M, O’Neill LA,Lawrence JB, Moore MJ, Caffrey DR, Fitzgerald KA: LincRNA-Cox2 is a long noncoding RNA induced by TLRs that mediates both activation and repression of immune response genes. Cytokine 2013;63:251-251.

37 Bond AM, VanGompel MJW, Sametsky EA, Clark MF, Savage JC, Disterhoft JF, Kohtz JD: Balanced gene regulation by an embryonic brain ncRNA is critical for adult hippocampal GABA circuitry. Nat Neurosci 2009;12:1020-1027.

-38 Li JQ Wu B, Xu J, Liu C: Genome-Wide Identification and Characterization of Long Intergenic Non-Coding RNAs in Ganoderma lucidum. Plos One 2014;9:e99442.

-39 Rosadini CV, Kagan JC: Early innate immune responses to bacterial LPS. Curr Opin Immunol 2017;44:14-19

40 Strasser A, Jost PJ, Nagata S: The many roles of FAS receptor signaling in the immune system. Immunity 2009;30:180-192. 